\title{
CHARACTERIZATION OF EXTRAGALACTIC 24 MICRON SOURCES IN THE SPITZER FIRST LOOK SURVEY
}

\author{
Lin Yan, ${ }^{1}$ George Helou, ${ }^{1}$ D. Fadda, ${ }^{1}$ F. R. Marleau, ${ }^{1}$ M. Lacy,${ }^{1}$ G. Wilson, ${ }^{1}$ B. T. Soifer, ${ }^{1,2}$ I. Drozdovsky, ${ }^{1}$ \\ F. Masci,${ }^{1}$ L. Armus,${ }^{1}$ H. I. Teplitz,${ }^{1}$ D. T. Frayer, ${ }^{1}$ J. Surace, ${ }^{1}$ L. J. Storrie-Lombardi, ${ }^{1}$ P. N. Appleton, ${ }^{1}$ \\ S. Chapman, ${ }^{2}$ P. Choi, ${ }^{1}$ F. Fan, ${ }^{1}$ I. Heinrichsen, ${ }^{1}$ M. Im, ${ }^{3}$ M. Schmitz, ${ }^{4}$ D. L. Shupe, ${ }^{1}$ and G. K. SQuires ${ }^{1}$ \\ Received 2004 April 3; accepted 2004 May 12
}

\begin{abstract}
In this paper, we present the initial characterization of extragalactic $24 \mu \mathrm{m}$ sources in the Spitzer First Look Survey by examining their counterparts at the $8 \mu \mathrm{m}$ and $R$ bands. The color-color diagram of $24 / 8 \mu \mathrm{m}$ versus $24 / 0.7 \mu \mathrm{m}$ is populated with 18,734 sources brighter than the $3 \sigma$ flux limit of $110 \mu \mathrm{Jy}$. The data cover a total area of $3.7 \mathrm{deg}^{2}$. The $24 / 0.7 \mu \mathrm{m}$ colors of these sources span almost 4 orders of magnitudes, while the $24 / 8 \mu \mathrm{m}$ colors are distributed over at least 2 orders of magnitude. In addition to identifying $\sim 30 \%$ of the total sample with infrared-quiescent, mostly low-redshift galaxies, we also found that (1) $23 \%$ of the $24 \mu \mathrm{m}$ sources $\left(\sim 1200 \mathrm{deg}^{-2}\right)$ with $\log \left[\nu f_{\nu}(24 \mu \mathrm{m}) / \nu f_{\nu}(8 \mu \mathrm{m})\right] \geq 0.3$ and $\log \left[\nu f_{\nu}(24 \mu \mathrm{m}) / \nu f_{\nu}(0.7 \mu \mathrm{m})\right] \geq 1.0$ are probably infrared luminous starburst galaxies with $L_{\mathrm{IR}} \geq 3 \times 10^{11} L_{\odot}$ at $z \geq 1$. In particular, $13 \%$ of the sample $\left(660 \mathrm{deg}^{-2}\right)$ are detected only at $24 \mu \mathrm{m}$, with no detectable emission in either the $8 \mu \mathrm{m}$ band or the $R$ band. With such extremely red IR/visible and mid-IR colors, these sources are good candidates for being ultraluminous infrared galaxies at $z \geq 2$. (2) $2 \%$ of the sample $\left(85 \mathrm{deg}^{-2}\right)$ have extremely red mid-infrared-to-optical color $\left(\log \left[\nu f_{\nu}(24 \mu \mathrm{m}) / \nu f_{\nu}(0.7 \mu \mathrm{m})\right] \geq 1.5\right)$ and fairly moderate $24 / 8 \mu \mathrm{m}$ color $\left(\log \left[\nu f_{\nu}(24 \mu \mathrm{m}) / \nu f_{\nu}(8 \mu \mathrm{m})\right] \sim 0.5\right)$, and they are likely candidates for being dust-reddened active galactic nuclei, like Mrk 231 at $z \sim 0.6-3$. (3) We anticipate that some of these sources with extremely red colors may be new types of sources, since they cannot be modeled with any familiar type of spectral energy distribution. We find that close to $38 \%$ of the $24 \mu \mathrm{m}$ sources have optical $R$ fainter than 23.0 Vega magnitudes, and $17 \%$ of these have no detectable optical counterparts brighter than the $R$ limit of $25.5 \mathrm{mag}$. Optical spectroscopy of these extremely faint $24 \mu \mathrm{m}$ sources is very difficult, and therefore mid-infrared spectroscopy from the Spitzer is critical for understanding their physical nature.
\end{abstract}

Subject headings: galaxies: active — galaxies: high-redshift — galaxies: starburst — infrared: galaxies — surveys

\section{INTRODUCTION}

The far-IR background detected by $C O B E$ (Puget et al. 1996; Fixsen et al. 1998) peaks at around $\sim 200 \mu \mathrm{m}$ with energy comparable to the optical/UV background. This implies that $50 \%$ of the integrated rest-frame optical/UV emission is thermally reprocessed by dust and radiated at mid- to far-infrared. Thus, dust-enshrouded galaxies with high IR/ visible ratios, particularly ultraluminous infrared galaxies $\left(L_{\mathrm{FIR}}>10^{12} L_{\odot}, L_{\mathrm{FIR}} / L_{\mathrm{vis}}>\right.$ a few $)$, make a significant contribution to the total energy budget and star formation over the history of the universe. Deep surveys based on the rest-frame UV/optical do not provide a complete census of galaxy populations, and their measurements of luminosity and star formation rate are only lower limits (Meuer et al. 1999; Yan et al. 1999). Deep Infrared Space Observatory (ISO) and submillimeter SCUBA observations have shown that the integrated luminosity densities from dusty sources peak at around $z \sim 1$, roughly a factor of 4-5 higher than those measured from the

\footnotetext{
${ }^{1}$ Spitzer Science Center, California Institute of Technology, 1200 East California Boulevard, MC 220-6, Pasadena, CA 91125; lyan@ipac.caltech.edu.

${ }^{2}$ California Institute of Technology Optical Observatories, Pasadena, CA 91125 .

${ }^{3}$ School of Earth and Environmental Sciences, Seoul National University, Shillim-dong, Kwanak-gu, Seoul 151-742, South Korea.

${ }^{4}$ IPAC, California Institute of Technology, MC 100-22, Pasadena, CA 91125 .
}

rest-frame optical surveys (Serjeant et al. 2000; Elbaz et al. 1999; Gruppioni et al. 2003; Blain et al. 1999). This peak in the star formation rate appears to be relatively flat out to $z \sim 2-4$ (Franceschini et al. 2001, 2002; Elbaz et al. 2002; Lagache et al. 2003).

While ISO deep surveys probe primarily galaxies at $z<1$ (Genzel \& Cesarsky 2000), and submillimeter SCUBA observations are limited to a small number of high-luminosity dusty sources at $z>2$ (Chapman et al. 2003), the dusty universe at $z>1$ is largely unexplored by systematic surveys in the mid-to-far-infrared wavelength. The $24 \mu \mathrm{m}$ imaging camera on the Spitzer Space Telescope (Werner et al. 2004) provides us with the first opportunity to do this. IRAS and ISO studies have shown that mid-infrared emission is a good indicator of the bolometric IR luminosity (Soifer et al. 1987; Surace 1998; Elbaz et al. 2002; Chary \& Elbaz 2001). At $0.7<z<2.5$, the $24 \mu \mathrm{m}$ band samples redshifted, rest-frame 6-12 $\mu \mathrm{m}$ emission from polycyclic aromatic hydrocarbons and very small dust grains in dusty galaxies, making it the most sensitive window to probe the high-redshift infraredbright galaxies.

In this paper, we make the first attempt to characterize the properties of $24 \mu \mathrm{m}$-selected galaxies within the Spitzer First Look Survey (FLS). ${ }^{5}$ We study the distribution in the $24 / 8$ and

\footnotetext{
${ }^{5}$ See http:// ssc.spitzer.caltech.edu/fls for details.
} 
TABLE 1

Summary of Optical, IRAC $8 \mu \mathrm{m}$, and MIPS $24 \mu \mathrm{m}$ Observations

\begin{tabular}{|c|c|c|c|}
\hline Parameter & Optical & IRAC $8 \mu \mathrm{m}$ & MIPS $24 \mu \mathrm{m}$ \\
\hline$\lambda_{\text {cent }}(\mu \mathrm{m})$ & 0.7 & 8.0 & 24.0 \\
\hline FWHM $(\operatorname{arcsec})$ & 1.0 & 2.2 & 5.5 \\
\hline Flux Limits......................................... & $25.5 \mathrm{mag}^{\mathrm{a}}$ & $20 \mu \mathrm{Jy}^{\mathrm{a}}$ & $110 \mu \mathrm{Jy}^{\mathrm{a}}$ \\
\hline$\nu f_{\nu}(\lim )\left(\operatorname{ergs~s}^{-1} \mathrm{~cm}^{-2}\right) \ldots \ldots \ldots \ldots$ & $1.3 \mathrm{E}-15^{\mathrm{b}}$ & $3.75 \mathrm{E}-15$ & $24 \mathrm{E}-15$ \\
\hline$\langle$ Exp. time $\rangle(\mathrm{s})$ & 1800 & 60 & 80 \\
\hline
\end{tabular}

${ }^{\text {a }}$ Here $R$ of $25.5 \mathrm{mag}$ is a $2 \sigma$ limit within a $3^{\prime \prime}$ diameter aperture. The $8 \mu \mathrm{m}$ flux $20 \mu \mathrm{Jy}$ is a $2 \sigma$ limit, including aperture correction. The $24 \mu \mathrm{m}$ flux $110 \mu \mathrm{Jy}$ is a flux cutoff, in which the $\mathrm{S} / \mathrm{N}$ at the peak pixel is greater than or equal to $3 \sigma$. See the text for details.

b $f_{R}(0 \mathrm{mag})=2780 \mathrm{Jy}(\mathrm{G}$. Neugebauer 1997, private communication).

$24 / 0.7 \mu \mathrm{m}[R(24,8) \text { and } R(24,0.7)]^{6}$ color-color diagram of $18,73424 \mu \mathrm{m}$-selected sources over an area of $3.7 \mathrm{deg}^{2}$. The $R(24,8)$ and $R(24,0.7)$ colors are indicative of the intrinsic slopes of the spectral energy distribution (SED) as well as indicative of the dust extinction and $K$-corrections. We pay special attention to the populations with extremely red $24 / 8$ and $24 / 0.7 \mu \mathrm{m}$ colors and estimate their surface densities. Throughout this paper, we adopt $H_{0}=70 \mathrm{~km} \mathrm{~s}^{-1}$ $\mathrm{Mpc}^{-1}, \Omega_{M}=0.3$, and $\Omega_{\Lambda}=0.7$. The magnitude system is in Vega.

\section{OBSERVATIONS AND DATA REDUCTION}

\subsection{Image Reduction and Source Extraction}

Table 1 summarizes all of the data that went into the analyses in this paper. Specifically, the $24 \mu \mathrm{m}$ flux cutoff of $110 \mu \mathrm{Jy}$ is for a signal-to-noise ratio $(\mathrm{S} / \mathrm{N})$ of $3 \sigma$, which is computed at the peak pixel. This is a conservative measurement in comparison with the $\mathrm{S} / \mathrm{N}$ calculated through a large aperture. After the reliable sources were selected with this conservative flux limit, we can use a lower S/N $(2 \sigma)$ to estimate the flux limits for sources not detected in the $8 \mu \mathrm{m}$ band or the $R$ band. The Spitzer data were taken with the Infrared Array Camera (IRAC) and the Multiband Imaging Photometer (MIPS; Fazio et al. 2004; Rieke et al. 2004). The 8 and $24 \mu \mathrm{m}$ data cover an area of $3.7 \mathrm{deg}^{2}$, where these two data sets overlap. Both IRAC and MIPS raw data were processed and mosaicked together by the pipeline provided by the Spitzer Science Center. Additional corrections to the basic calibrated data images were included; see Lacy et al. (2004) for details. The $8 \mu \mathrm{m}$ source catalog is generated using SExtractor (Bertin \& Arnout 1996). We used the photometry within a 6". 1 diameter aperture. The total fluxes are obtained by applying the appropriate aperture corrections, which are scaled to the $24^{\prime \prime}$ diameter aperture. This is at most around $30 \%$ (Lacy et al. 2004). The $24 \mu \mathrm{m}$ source catalog was generated using StarFinder (Diolaiti et al. 2000). The aperture correction for obtaining the total $24 \mu \mathrm{m}$ flux is $\sim 10 \%$. The $R$-band images were taken with the MOSAIC- 1 camera on the $4 \mathrm{~m}$ telescope at the Kitt Peak National Observatory. The reduced and stacked images as well as the source catalogs have been publicly released, and the detailed description of the observations, data reduction, and catalogs can be found in Fadda et al. (2004).

\subsection{Band-merged Catalog}

We cross-identified the $24 \mu \mathrm{m}$ sources in the $8 \mu \mathrm{m}$ and $R$ band by simple positional matching. The IRAC astrometry was fine-tuned using the reference positions from the 2MASS point sources. The MIPS $24 \mu \mathrm{m}$ images were aligned with the VLA $20 \mathrm{~cm}$ radio positions. The mean positional differences between $R$ and $24 \mu \mathrm{m}$ for bright and unsaturated sources are $00^{\prime \prime} 1$ with an rms of 0.4 in both right ascension and declination. Similarly, we found that $\langle\Delta$ R.A. $\rangle=0 . " 04 \pm 0 . " 6$ and $\langle\Delta$ decl. $\rangle=$ 0 "'03 \pm 0 "' 6 between the IRAC $8 \mu \mathrm{m}$ and $R$-band images. We use $2^{\prime \prime}$ matching radii, generously chosen to allow somewhat larger astrometric uncertainties for fainter sources. Multiple matches between the 24 and $8 \mu \mathrm{m}$ catalogs are neglible (less than $0.05 \%$ ). The fraction of multiple matches between the $24 \mu \mathrm{m}-$ and $R$-band catalogs is higher, about $7 \%$. In these cases, we choose the closest matches in the positional centroids between the two bands. Stellar contamination for a $24 \mu \mathrm{m}-$ selected sample is expected to be low since we are sampling the tail of the Rayleigh-Jeans energy distribution and since the FLS Galactic latitude is $37^{\circ}$. Stars brighter than $R$ of 20 mag can be easily identified on the $R$-band images. For sources with $20<R<23$, we used the stellarity index ( $>0.8$ sources are stars) measured with SExtractor. The total stellar contamination is estimated to be only $7.5 \%$. We have visually spotchecked the final catalog on the image display.

\section{RESULTS}

We have a total of 18,734 sources, which are detected at $24 \mu \mathrm{m}$ with fluxes brighter than the $3 \sigma$ flux limit of $110 \mu \mathrm{Jy}$. Of these, close to half of them have good detections in all three bands. The remaining half of the $24 \mu \mathrm{m}$ sources have no detections in one or both of the $8 \mu \mathrm{m}$ and $R$ bands. Figure 1 (Plate 1) gives the two-dimensional stamp images of a set of representative examples. As shown, some bright $24 \mu \mathrm{m}$ sources have no counterparts at either the $8 \mu \mathrm{m}$ band or the $R$ band. These cases will be discussed in detail below.

The main result of this paper is presented in Figure $2 a$ (Plate 2), showing the $24 / 8$ and $24 / 0.7 \mu \mathrm{m}$ color-color distribution of our sources. Figure $2 b$ presents the expected colorcolor tracks as a function of redshifts computed from known types of SED templates. Figure $2 a$ shows a broad correlation between higher IR/visible ratios and IR colors, indicative of more intensely heated dust, similar to the trends seen in the IRAS data (Soifer \& Neugebauer 1991). Using this figure, we can crudely classify various types of $24 \mu \mathrm{m}$-selected sources by comparison with the expected colors of known SED templates as well as with known objects in the FLS region. The SED templates include starburst galaxy Arp 220 and M82 (Silva et al. 1998; Chary \& Elbaz 2001), NGC 253 (Fadda et al. 2002), a radio-quiet QSO, normal spiral galaxy M51, and dust-reddened active galactic nucleus (AGN) NGC 1068 (Dale et al. 2001; Dale \& Helou 2002). To show the colors of an early-type galaxy, we construct the SED for the bulge of M31 within an aperture of $4^{\prime}$ diameter using the near-IR and IRAS data published in Soifer et al. (1986). The optical part of the SED for the M31 bulge was taken as a 10 Gyr old elliptical SED from Bruzual \& Charlot, ${ }^{7}$ then scaled and matched with the infrared part to produce a full SED covering from 0.1 to $100 \mu \mathrm{m}$.

\footnotetext{
${ }^{7}$ See ftp://gemini.tuc.noao.edu/pub/charlot/bc5.
}

\footnotetext{
${ }^{6}$ Here $\quad R(24,8) \equiv \log \left[\nu f_{\nu}(24 \mu \mathrm{m}) / \nu f_{\nu}(8 \mu \mathrm{m})\right], \quad$ and $\quad R(24,0.7) \equiv$ $\log \left[\nu f_{\nu}(24 \mu \mathrm{m}) / \nu f_{\nu}(0.7 \mu \mathrm{m})\right]$.
} 

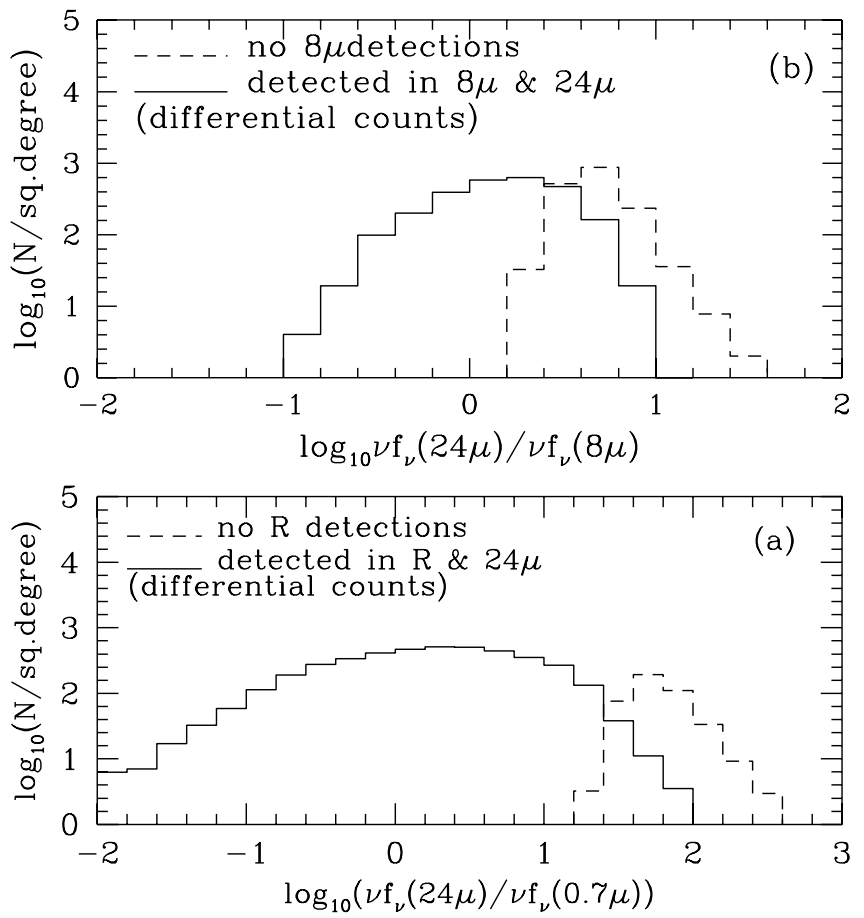

FIG. 3. - The $24 \mu \mathrm{m}$ source counts as functions of $R(24,0.7)$ and $R(24,8)$. The dashed lines in both panels are sources with flux limits in either the $8 \mu \mathrm{m}$ or the $R$ band. The number counts are differential, and the $y$-axis has units of numbers of sources per square degree. The total area coverage is $3.7 \mathrm{deg}^{2}$, and the total number of sources is 18,734 .

\subsection{Infrared Quiescent Sources}

Comparing Figures $2 a$ and $2 b$, the color-color distribution can be crudely classified into two extreme regions - the infrared quiescent region where $R(24,8) \leq 0.5$ and $R(24,0.7) \leq 0$, and the infrared luminous region with $R(24,8)>0.5$ and $R(24,0.7)>1$. The region between these two probably contains a mixture of starbursts and normal galaxies at various redshifts. In Figure $2 a$, the black points occupy most of the first region. These are $24 \mu \mathrm{m}$ sources that have significant detections at $8 \mu \mathrm{m}$ as well as at the $R$ band. Comparing with the model color-color tracks, we infer that the sources with $R(24,8) \leq 0.5$ and $R(24,0.7) \leq 0$ are primarily normal, infrared-faint galaxies at low redshift $(z<0.7)$ and optically selected QSOs with a wider redshift distribution. Figures $3 a$ and $3 b$ show the differential number counts as functions of colors, $R(24,8)$ and $R(24,0.7)$. A little less than $30 \%$ of the total population are infrared quiescent spiral galaxies and early-type galaxies at low redshifts as well as optically selected QSOs. The galaxies with extremely low $24 / 8 \mu \mathrm{m}$ flux ratios are old bulges, such as M31. Their colors cannot be explained by pure interstellar dust emission, and they must have substantial stellar photospheric and dust envelope contributions coming into the $8 \mu \mathrm{m}$ band.

To test this interpretation of Figure $2 a$ further, we have used the NASA/IPAC Extragalactic Database and identified the sources in our catalog with the low-redshift galaxies (magenta triangles) and optically selected QSOs (cyan triangles) from the Sloan Digital Sky Survey (SDSS). ${ }^{8}$ The locations of these sources with known types and redshifts in Figure $2 a$ generally confirm our interpretation. The cyan points in Figure $2 a$ can

\footnotetext{
${ }^{8}$ See http://www.sdss.org.
}

be well explained by the computed colors of a radio-quiet QSO SED template in Figure $2 b$. These optically selected QSOs have fairly flat SEDs (Neugebauer et al. 1987; Sanders et al. 1989); therefore, their colors are around $R(24,8) \sim 0$ and $R(24,0.7) \sim 0$ with very little change in redshifts. One specific example is an SDSS QSO at $z=4.5445$, with fluxes of 744,98 , and $27 \mu \mathrm{Jy}$ at 24,8 , and $0.7 \mu \mathrm{m}$, respectively; thus $R(24,8)=0.4$ and $R(24,0.7)=-0.1$.

\subsection{The Nature of the Extreme $24 \mu \mathrm{m}$ Populations}

Figure $2 a$ illustrates the colors of four types of sources marked with small black, red, green, and blue points. The objects indicated with black points have been discussed before. The red points consist of $31 \%$ of the $24 \mu \mathrm{m}$ sources, which are detected only in the $R$ band, not at the $8 \mu \mathrm{m}$ band. The green points represent $4 \%$, with detections only at $8 \mu \mathrm{m}$, but no counterparts in $R$ brighter than the $2 \sigma$ limit of $25.5 \mathrm{mag}$. Finally, $13 \%$ of the sample (660 objects $\mathrm{deg}^{-2}$ ) are not detected in either $8 \mu \mathrm{m}$ or $R$, and they are shown as small blue points in Figure $2 a$. The mean fluxes of the objects marked as red, green, and blue points are roughly between 230 and $360 \mu \mathrm{Jy}$. Some of the more interesting sources revealed by the $24 \mu \mathrm{m}$ images are the ones that have $R(24,0.7)>1$ and $R(24,8)>0.3$ in Figure $2 a$. These sources are candidates for being luminous starburst galaxies at $z \geq 1$. Using this color-color cut, we estimate that $23 \%\left(\sim 1200 \mathrm{deg}^{-1}\right)$ of our $24 \mu \mathrm{m}$ sample are such objects. In particular, those sources detected only at $24 \mu \mathrm{m}$ (blue points) are likely to be ultraluminous infrared galaxies at $z>2\left(660 \mathrm{deg}^{-2}\right)$. At $z=1$, our $3 \sigma 24 \mu \mathrm{m}$ flux limit of $110 \mu \mathrm{Jy}$ corresponds to the observed luminosity $\nu L_{\nu}$ of $2 \times 10^{10} L_{\odot}$. If we ignore the filter difference between the Spitzer $24 \mu \mathrm{m}$ and the IRAS $12 \mu \mathrm{m}$ (the $24 \mu \mathrm{m}$ bandwidth is about half of the $12 \mu \mathrm{m}$ filter), the $z=1$ observed $24 \mu \mathrm{m}$ luminosity is roughly the rest-frame IRAS $12 \mu \mathrm{m}$ luminosity.

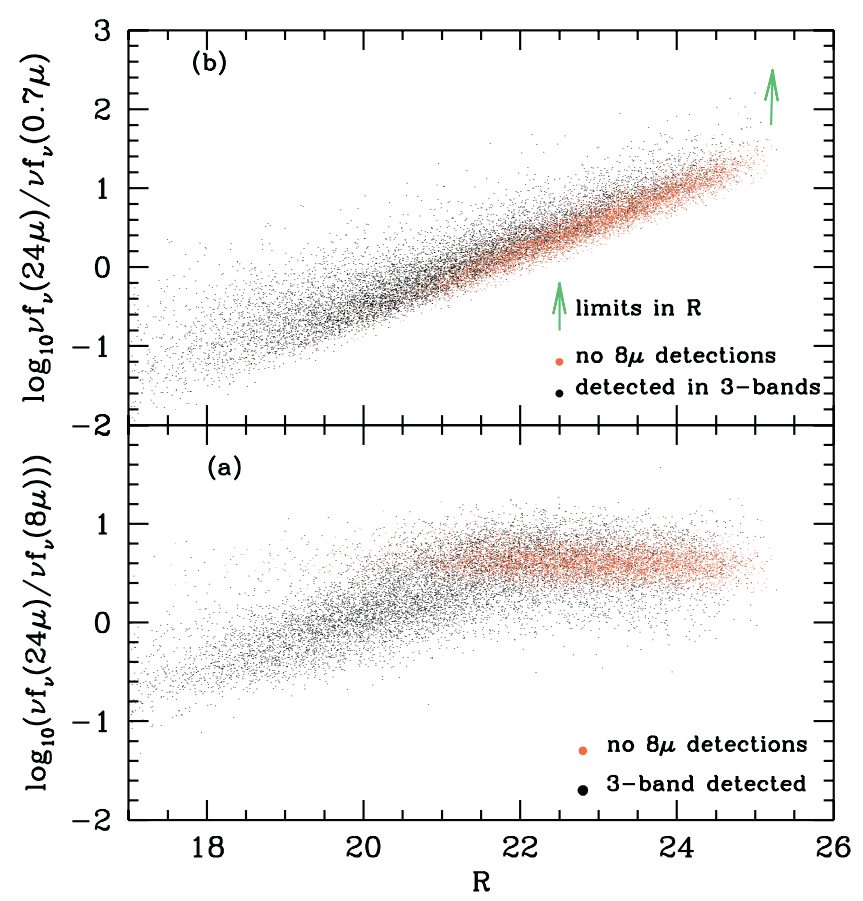

FIG. 4.- (a) $24 / 8 \mu \mathrm{m}$ flux ratio vs. $R$ magnitude for the $24 \mu \mathrm{m}-$ selected sample. (b) Color-magnitude diagram of the $24 / 0.7 \mu \mathrm{m}$ flux ratios vs. $R$ magnitude. 
The total infrared luminosity $L_{\mathrm{IR}}(8-1000 \mu \mathrm{m})$ is correlated with the IRAS $12 \mu \mathrm{m}$ luminosity $\nu L_{\nu}(12 \mu \mathrm{m})$, in the form of $L_{\mathrm{IR}}=0.89 \nu L_{\nu}(12 \mu \mathrm{m})^{1.094} L_{\odot}$, as derived from the IRAS Revised Bright Galaxy Sample (Soifer et al. 1987; Chary \& Elbaz 2001). This implies that the $z>1$ starbursts should have $L_{\mathrm{IR}} \geq 2.7 \times 10^{11} L_{\odot}$. As suggested by the $24 \mu \mathrm{m}$ source counts (Marleau et al. 2004), the FLS data may reach a $z \sim 2$ starburst population. Our flux limit implies that at $z \sim 2$, the infrared luminosity $L_{\mathrm{IR}}$ should be brighter than $2 \times 10^{12} L_{\odot}$.

Another interesting population of sources are those with $R(24,0.7)>1.5$ but with a fairly constant $R(24,8) \sim 0.5$. Figure $2 b$ suggests that these sources are likely to be heavily dust-reddened AGNs like NGC 1068 or like Mrk 231 at $z>1$. These sources could be separated from the general population using the IRAC $8 / 4.5$ and $5.8 / 3.5 \mu \mathrm{m}$ color-color selection (Lacy et al. 2004). The surface density of these dusty AGNs are on the order of $\sim 85 \mathrm{deg}^{-2}$, and they constitute $\sim 2 \%$ of the total $24 \mu \mathrm{m}$ population. To confirm our prediction that we can use $R(24,8)$ versus $R(24,0.70)$ to select high-redshift, infrared luminous galaxies, we mark on Figure $2 a$ the several $24 \mu \mathrm{m}$ sources detected with the SCUBA at $850 \mu \mathrm{m}$ (see Frayer et al. 2004 for detail). These SCUBA sources could be at $z \sim 1-3$, as shown in Chapman et al. (2003). Our computed color-color tracks in Figure 2 illustrate in a broad sense what range of colors each type of source should have. A small number of sources with extremely red $24 / 8 \mu \mathrm{m}$ and $24 \mu \mathrm{m} / R$ colors cannot be explained completely by models with known types of SEDs. This could be suggestive of potentially new classes of objects.
We examine the optical brightness of $24 \mu \mathrm{m}$-selected sources in Figure 4 , showing $R(24,0.7)$ and $R(24,8)$ as functions of $R$ magnitude for the $24 \mu \mathrm{m}$ sources. Close to $36 \%$ of the $24 \mu \mathrm{m}$ sources have $R$ magnitudes fainter than $23.0 \mathrm{mag}$. Such a faint optical magnitude might suggest that they are at $z>0.5$, supported by the measurements from optical $R$-band-selected redshift surveys (Lilly et al. 1996; Cohen 2001). Many of these sources are well within the brightness regime of the $10 \mathrm{~m}$ class telescopes for obtaining optical spectroscopic redshifts. However, the $24 \mu \mathrm{m}$ sources without $R$ counterparts fainter than $2 \sigma$ of $25.5 \mathrm{mag}$ will be difficult to follow up with optical spectroscopy. The redshifts and physical natures of these sources could be measured using mid-infrared spectroscopy from the Infrared Spectrograph for Spitzer (IRS). Particularly, for sources with $24 \mu \mathrm{m}$ fluxes brighter than $750 \mu \mathrm{Jy}$, the IRS would be sensitive enough to obtain good low-resolution $\mathrm{S} / \mathrm{N}$ spectra covering $5 / 38 \mu \mathrm{m}$ with a total of $1-2 \mathrm{hr}$ of integration. We have a total of 46 sources that have $24 \mu \mathrm{m}$ fluxes brighter than $750 \mu \mathrm{Jy}$ and an $R$-band magnitude fainter than $25.0 \mathrm{mag}$.

This work is based in part on observations made with the Spitzer Space Telescope, which is operated by the Jet Propulsion Laboratory, California Institute of Technology, under NASA contract 1407. Support for this work was provided by NASA. We also made use of the NASA/IPAC Extragalactic Database (NED), which is operated by the Jet Propulsion Laboratory, California Institute of Technology, under contract with the National Aeronautics and Space Administration.
Bertin, E., \& Arnouts, S. 1996, A\&AS, 117, 393

Blain, A. W., Smail, I., Ivison, R. J., \& Kneib, J.-P. 1999, MNRAS, 302, 632 Chapman, S. C., Blain, A. W., Ivison, R. J., \& Smail, I. R. 2003, Nature, 422, 695

Chary, R., \& Elbaz, D. 2001, ApJ, 556, 562

Cohen, J. 2001, AJ, 121, 2895

Dale, D. A., \& Helou, G. 2002, ApJ, 576, 159

Dale, D. A., Helou, G., Contursi, A., Sibermann, N. A., \& Kolhatkar, S. 2001, ApJ, 549, 215

Diolaiti, E., Bendinelli, O., Bonaccini, D., Close, L. M., Currie, D. G., \& Parmeggiani, G. 2000, Proc. SPIE, 4007, 879

Elbaz, D., Cesarsky, C. J., Chanial, P., Aussel, H., Franceschini, A., Fadda, D., \& Chary, R. R. 2002, A\&A, 384, 848

Elbaz, D. et al. 1999, A\&A, 351, L37

Fadda, D., Flores, H., Hasinger, G., Franceschini, A., Altieri, B., Cesarsky, C. J., Elbaz, D., \& Ferrando, Ph. 2002, A\&A, 383, 838

Fadda, D., Jannuzi, B., Ford, A., \& Storrie-Lombardi, L. J. 2004, AJ, 128, 1

Fazio, G., et al. 2004, ApJS, 154, 10

Fixsen, D. J., Dwek, E., Mather, J. C., Bennett, C. L., \& Shafer, R. A. 1998, ApJ, 508, 123

Franceschini, A., Aussel, H., Cesarsky, C. J., Elbaz, D., \& Fadda, D. 2001, A\&A, 378, 1

Franceschini, A., Fadda, D., Cesarsky, C. J., Elbaz, D., Flores, H., \& Granato, G. L. 2002, ApJ, 568, 470

Frayer, D., et al. 2004, ApJS, 154, 137

Genzel, R., \& Cesarsky, C. J. 2000, ARA\&A, 38, 761
Gruppioni, C., Pozzi, F., Zamorani, G., Ciliegi, P., Lari, C., Calabrese, E., La Franca, F., \& Matute, I. 2003, MNRAS, 341, L1

Lacy, M., et al. 2004, ApJS, 154, 166

Lagache, G., Dole, H., \& Puget, J.-L. 2003, MNRAS, 338, 555

Lilly, S. J., Le Fèvre, Hammer, F., \& Crampton, D. 1996, ApJ, 460, L1

Marleau, F., et al. 2004, ApJS, 154, 66

Meuer, G. R., Heckman, T. M., \& Calzetti, D. 1999, ApJ, 521, 64

Neugebauer, G., Green, R. F., Matthews, K., Schmidt, M., Soifer, B. T., \& Bennett, J. 1987, ApJS, 63, 615

Puget, J.-L., Abergel, A., Bernard, J.-P., Boulanger, F., Burton, W. B., Desert, F.-X., \& Hartmann, D. 1996, A\&A, 308, L5

Rieke, G., et al. 2004, ApJS, 154, 25

Sanders, D. B., Phinney, E. S., Neugebauer, G., Soifer, B. T., \& Matthews, K. 1989, ApJ, 347, 29

Serjeant, S., Mobasher, B., Gruppioni, C., \& Oliver, S. 2000, MNRAS, 317, L29

Silva, L., Granato, G. L., Bressan, A., \& Danese, L. 1998, ApJ, 509, 103

Soifer, B. T., \& Neugebauer, G. 1991, AJ, 101, 354

Soifer, B. T., Rice, W. L., Mould, J. R., Gillett, F. C., Rowan-Robinson, M., \& Habing, H. J. 1986, ApJ, 304, 651

Soifer, B. T., et al. 1987, ApJ, 320, 238

Surace, J. 1998, Ph.D. thesis, Univ. Hawaii

Werner, M., et al. 2004, ApJS, 154, 1

Yan, L., McCarthy, P. J., Freudling, W., Teplitz, H. I., Malumuth, E. M., Weymann, R. J., \& Malkan, M. A. 1999, ApJ, 519, L47 

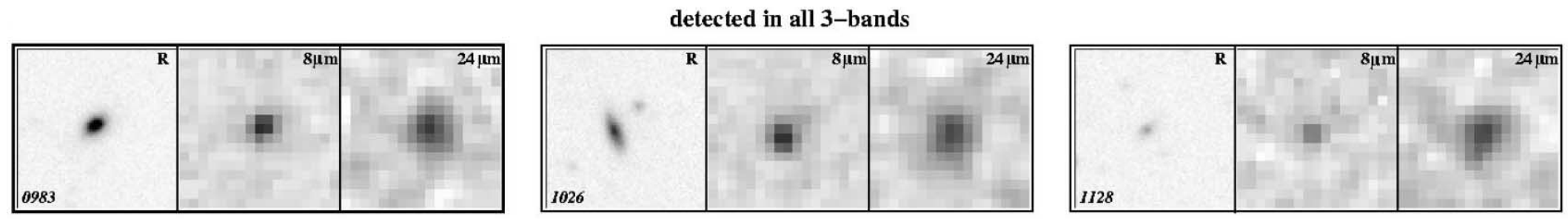

detected in $24 \mu \mathrm{m} \& \mathrm{R}$, not in $8 \mu \mathrm{m}$
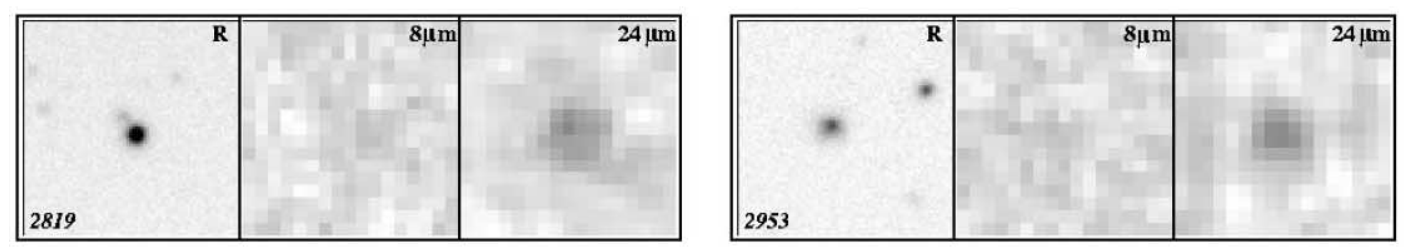

detected in $24 \mu \mathrm{m} \& 8 \mu \mathrm{m}$, not in $\mathbf{R}$
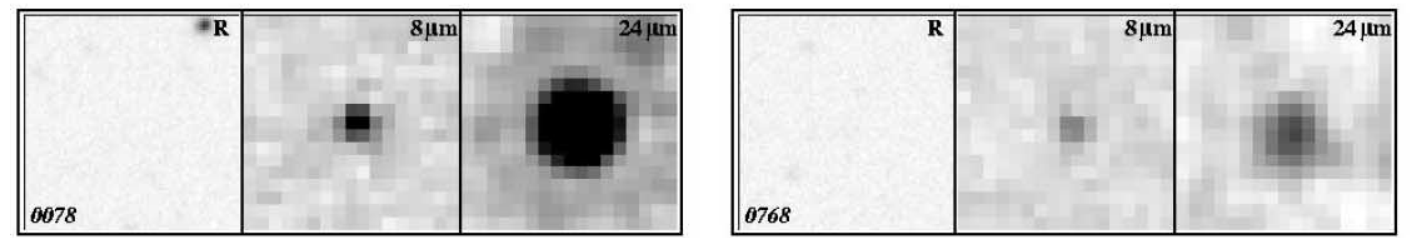

detected only in $24 \mu \mathrm{m}$, not in $8 \mu \mathrm{m} \& \mathrm{R}$
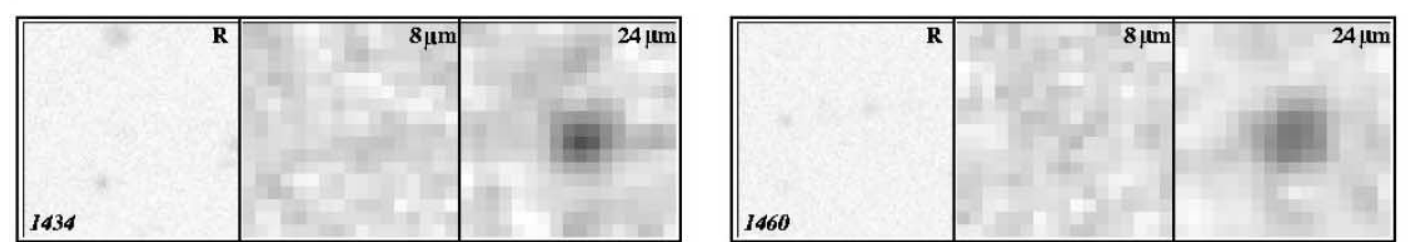

FIG. 1.-A few representative examples of four types of $24 \mu \mathrm{m}$ sources: sources detected in all three bands; sources detected in $8 \mu \mathrm{m}$ but not in $R$; sources detected in $R$ but not in $8 \mu \mathrm{m}$; and $24 \mu \mathrm{m}$-detected sources only.
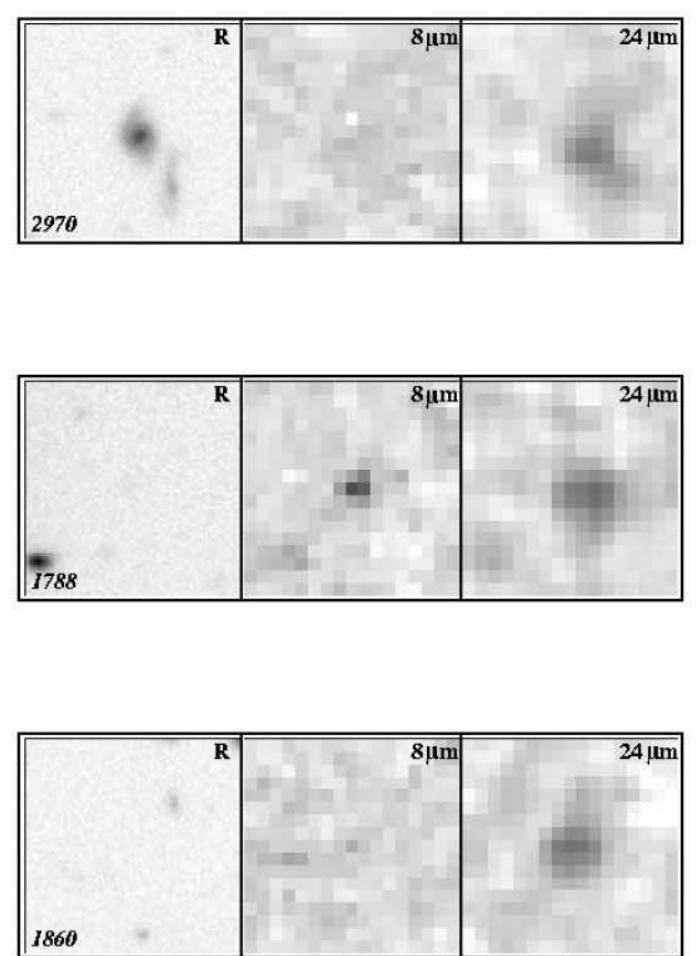


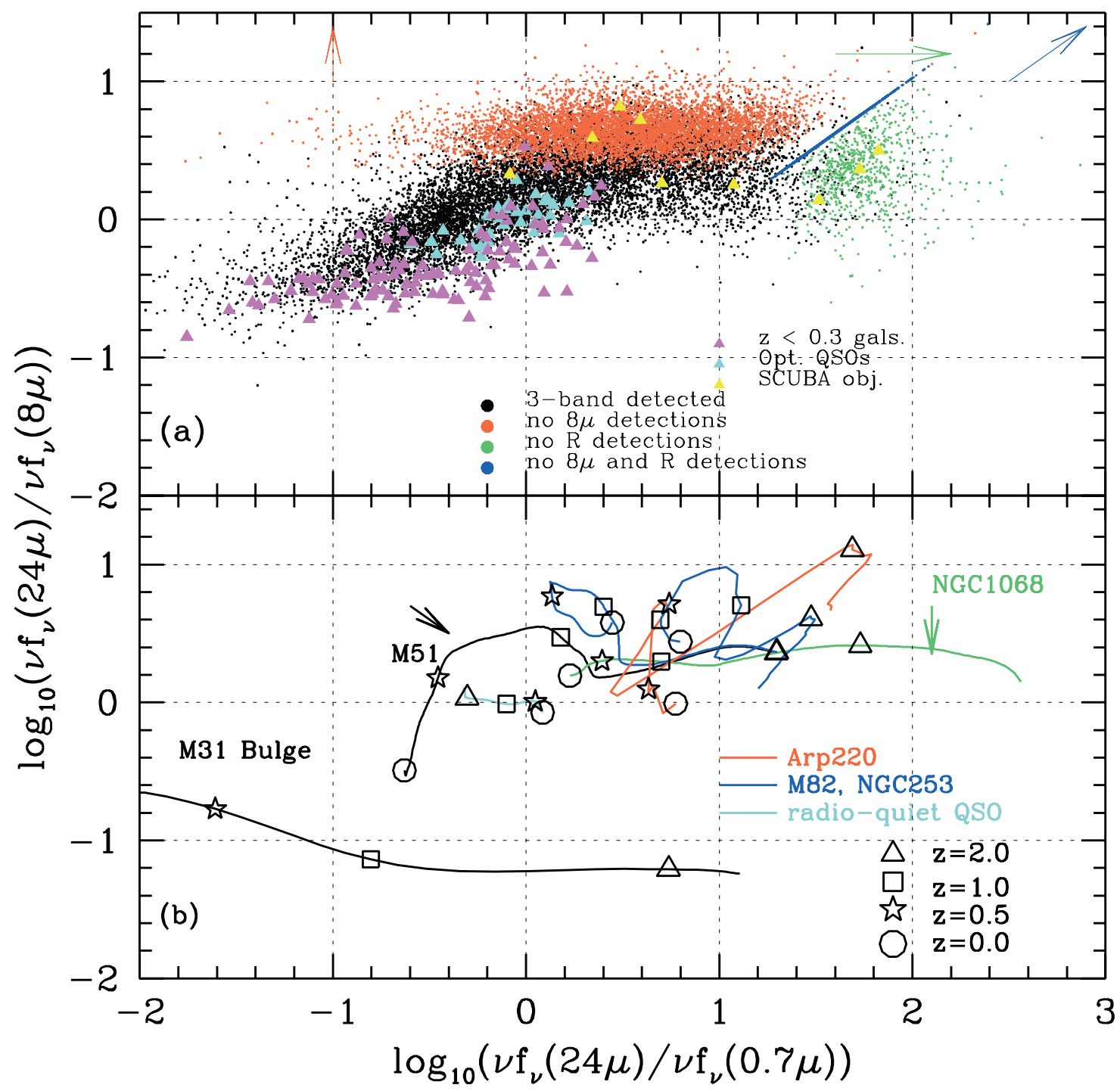

FIG. 2.-Color-color plot between $\log \left[\nu f_{\nu}(24 \mu \mathrm{m}) / \nu f_{\nu}(8 \mu \mathrm{m})\right]$ and $\log \left[\nu f_{\nu}(24 \mu \mathrm{m}) / \nu f_{\nu}(R)\right]$. (a) Data for a total of 18,734 sources selected at $24 \mu \mathrm{m}$ in the FLS main field. The small black points are sources detected in all three bands; the small green points are sources only detected in the $8 \mu \mathrm{m}$ bands and not detected in the optical $R$ band; the small red points indicate sources with significant detections in the $R$ band but no detections at $8 \mu$ m; the small blue points represents the sources detected only at $24 \mu \mathrm{m}$. To illustrate the lower limits in colors, we marked the arrows with a corresponding color code for the red, green, and blue sources. The big yellow triangles are sources with SCUBA detections; the cyan triangles are optically selected QSOs with known redshifts and spectra; and the magenta triangles are low-redshift galaxies identified from the SDSS. $(b)$ Expected color tracks as a function of redshifts computed from a set of SED templates for known local galaxies. 